\title{
Early evolution stage of AGN
}

\section{Kunert-Bajraszewska ${ }^{1}$, A. Labiano ${ }^{2}$, A. Siemiginowska ${ }^{3}$, M. Guainazzi ${ }^{4}$ and M. Gawroński ${ }^{1}$}

\author{
${ }^{1}$ Toruń Centre for Astronomy, Faculty of Physics, Astronomy and Informatics, NCU, \\ Grudziadzka 5 , 87-100 Toruń, Poland \\ email: magda@astro.uni.torun.pl
${ }^{2}$ ETH Zurich, Institute for Astronomy, Wolfgang-Paulistrasse 27, CH-8093 Zurich, Switzerland
${ }^{3}$ Harvard-Smithsonian Center for Astrophysics, 60 Garden St, Cambridge, USA
${ }^{4}$ ESA, European Space Astronomy Centre, 28691 Villanueva de la Canada, Madrid, Spain

\begin{abstract}
Radio sources are divided into two distinct morphological groups of objects: FanaroffRiley type I and type II sources. There is a relatively sharp luminosity boundary between these at low frequency. The nature of the FR division is still an open issue, as are the details of the evolutionary process in which younger and smaller GHz-peaked spectrum (GPS) and compact steep spectrum (CSS) sources become large-scale radio structures. It is still unclear whether FR II objects evolve to become FR Is, or whether a division has already occurred amongst CSS sources and some of these then become FR Is and some FR IIs. We explored evolution scenarios of AGNs using new radio, optical and X-ray data of unstudied so far Low Luminosity Compact (LLC) sources. We suggest that the determining factors of the further evolution of compact radio objects could occur at subgalactic (or even nuclear) scales, or they could be related to the radio jet - interstellar medium (ISM) interactions and evolution. Our studies show that the evolutionary track could be related to the interaction, strength of the radio source and excitation levels of the ionized gas instead of the radio morphology of the young radio source.
\end{abstract}

\section{Overview of the results}

\subsection{Radio observations}

Using Faint Images of the Radio Sky at Twenty cm (FIRST) and the Sloan Digital Sky Survey (SDSS) we have created a sample of 44 Low Luminosity Compact (LLC) sources and observed them with MERLIN at L-band and C-band. The selection criteria used for the new sample resulted in approximately one-third of the LLC sources having a value of $1.4 \mathrm{GHz}$ radio luminosity comparable to FR Is (Fanaroff \& Riley 1974). In radio power versus linear size diagram they occupy space below main evolutionary path (Kunert-Bajraszewska et al. 2010).

Most of the sources have been resolved, showing complex, asymmetric and breaking-up radio structures. We have suggested that some of them could be candidates for compact faders. We concluded that low luminosity young AGNs may develop to diffuse, large-scale structures as their weak jets are disrupted before escaping their host galaxies. They may undergo disrupted evolution many times as they are finally able to escape the host galaxy and evolve further as FR I or hybrid object (Kunert-Bajraszewska et al. 2010, Cegłowski et al. 2013).

\subsection{Spectral analysis}

We have also studied the optical properties of LLC sources as a group but also within a larger sample that also includes the published samples of CSS, GPS and FR I/FR II 
objects (Buttiglione et al. 2010). We have classified the LLC sources as high and low excitation galaxies (HEG and LEG respectively). The optical properties of LLC objects are, in general, consistent with brighter CSS and large-scale radio sources, although the LLC objects have lower values of [OIII] luminosity than the more powerful CSS sources. However, when LLC objects are added to the other samples, HEGs and LEGs seem to follow independent, parallel evolutionary tracks: $\mathbf{G P S}_{\mathbf{H E G}}-\mathbf{C S S}_{\mathbf{H E G}}-\mathbf{F R} \mathbf{I I}_{\mathbf{H E G}}$ and $\mathbf{G P S}_{\mathbf{L E G}}-$ $\mathbf{C S S}_{\text {LEG }}-\mathbf{F R}_{\text {LEG }}$.

Furthermore, the HEG/LEG classification for the LLC objects, bright CSSs and largescale sources is independent of radio power and size. Concerning the radio morphology, CSS sources behave like FR II objects at all radio powers, suggesting that the differences in the mode of accretion (or black hole spin) between LEG and HEG sources is already visible in the CSS phase. We could however, still be missing the precursors of the large FR I sources (Kunert-Bajraszewska \& Labiano 2010).

\subsection{X-ray properties}

Finally, we report first X-ray Chandra observations of seven LLC sources and use these data together with that available in the literature for strong CSS, GPS and FR I/FR II objects to study the relation between morphology, X-ray properties and excitation modes in radio-loud AGNs (Kunert-Bajraszewska et al. 2013, 2014). We found the following results:

- The HEG and LEG sources occupy distinct locus in radio/X-ray luminosity diagram, notwithstanding their evolutionary stage.

- The less radio powerful FR Is have higher radio/X-ray luminosity ratio than many FR IIs what may imply higher X-ray luminosity decrease with radio power in FR Is than FR IIs. This is in agreement with previously postulated different X-rays emission origins. In case of FR Is it is connected with the base of the jet while for FR IIs X-ray emission originates from accretion. The same can be true for compact sources as well. The results hints that below certain level of radio luminosity GPSs and CSSs starts to resemble FR Is.

\section{Acknowledgements}

This work was supported by the National Science Centre under grant DEC-2011/01/D/ST9/00378.

\section{References}

Buttiglione S., Capetti, A., Celotti, A., et al., 2010, A\&A, 509, 6

Cegłowski, M., Gawroński, M. P., \& Kunert-Bajraszewska, M., 2013, A\&A, 557, 75

Fanaroff, B. L. \& Riley, J. M. 1974, MNRAS 167, 31P

Kunert-Bajraszewska, M., Gawroński, M. P., Labiano, A., \& Siemiginowska, A. 2010, MNRAS, 408, 2261

Kunert-Bajraszewska, M. \& Labiano, A., 2010, MNRAS, 408, 2279

Kunert-Bajraszewska, M., Siemiginowska, A., \& Labiano, A., 2013, ApJ, 772, L7

Kunert-Bajraszewska, M., Labiano, A., Siemiginowska, A., \& Guainazzi, M. 2014, MNRAS, 437, 3063 